See discussions, stats, and author profiles for this publication at: https://www.researchgate.net/publication/331314369

\title{
Controlled Growth of Highly Aligned Cu Nanowires by Pulse Electrodeposition in Nanoporous Alumina
}

Article in Journal of Nanoscience and Nanotechnology · July 2019

Dol: 10.1166/jnn.2019.16868

CITATIONS

3 authors:

Arti Yadav

Newcastle University

3 PUBLICATIONS 3 CITATIONS

SEE PROFILE

Steve John Bull

Newcastle University

272 PUBLICATIONS 6,649 CITATIONS

SEE PROFILE

Some of the authors of this publication are also working on these related projects:

Project Nanomaterials and Pulse Deposition View project

Project Heating of droplets View project
Musuvathi S Bobji

Indian Institute of Science

67 PUBLICATIONS 893 CITATIONS

SEE PROFILE 


\title{
Controlled Growth of Highly Aligned Cu Nanowires by Pulse Electrodeposition in Nanoporous Alumina
}

\author{
Arti Yadav', M. S. Bobji², and Steve J. Bull1,* \\ ${ }^{1}$ School of Engineering, Newcastle University, Newcastle Upon Tyne, NE1 7RU, UK \\ ${ }^{2}$ Department of Mechanical Engineering, Indian Institute of Science, Bangalore 560012, India
}

\begin{abstract}
Metallic nanowire networks are emerging as potential replacements for transparent conducting oxide coatings because of their high conductivity, flexibility and relative transparency. However, a cheap, reliable and controlled manufacturing process is required to exploit this and the surface of the copper nanowire needs to be protected if high conductivity is to be retained. In this study a fabrication method for highly aligned and densely packed copper nanowires with controlled length using pulseelectrodeposition and a nanoporous alumina template has been developed. Nanoporous alumina was obtained by anodisation of pure aluminum in oxalic acid using a two-step anodisation process. In order to provide the conductivity at the bottom of the pores, a dendritic structure at the interface was created through the stepwise voltage reduction method with a voltage reduction rate of $15 \mathrm{~V} / \mathrm{s}$ followed by mild chemical etching. Highly repeatable near $100 \%$ filling of copper is achieved. Copper nanowire length was highly controllable from $100 \mathrm{~nm}$ to $2 \mu \mathrm{m}$ with a fixed diameter of $60 \pm 5 \mathrm{~nm}$ by monitoring current density during the deposition. Such controlled growth of $\mathrm{Cu}$ nanowires could lead towards transparent conducting layer applications but the protection of the material against oxidation remains an issue.
\end{abstract}

Keywords: Anodising, Nanoporous Alumina, Barrier Layer Thinning, Cu Nanowires.

\section{INTRODUCTION}

One dimensional nanostructures such as nanowires and nanorods have become a major subject of research over last few years because of their enormous potential applications in nanotechonogy. ${ }^{1-3}$ For instance, copper nanowires have recently been suggested as excellent candidates for transparent conducting films because $\mathrm{Cu}$ has high intrinsic conductivity; it is a potential alternative to indium tin oxide (ITO) and silver nanowires. In addition, $\mathrm{Cu}$ is only $6 \%$ less conductive than Ag, very abundant (1000 times more abundant than $\mathrm{Ag}$ ), and 100 times less expensive than $\mathrm{Ag}$ or ITO, ${ }^{4}$ and several studies have demonstrated how copper nanowire films can achieve high optical transmittance with high electrical conductivity ${ }^{5-7}$ However, there remain challenges for the passivation of the copper nanowires because they are prone to oxidation under ambient conditions, and thus they cannot retain performance comparable to that of ITO.

To date, a variety of methods have been developed to fabricate nanowires. ${ }^{1,2,8,9}$ Controlled nanowire geometry

\footnotetext{
*Author to whom correspondence should be addressed.
}

has generally been achieved by using lithography, ${ }^{8,9}$ however this technique is complex, expensive and requires specialized equipment with inherent limitations. ${ }^{1}$ The template-based approach is an alternate method to fabricate nanowires, ${ }^{10}$ and substrates such as mesoporous silica, patterned polycarbonate and nanoporous alumina are used to create the template. ${ }^{11-13}$ Porous alumina produced by anodisation of aluminium is often used as its geometry can be tuned. ${ }^{11,14}$ Despite this only a few studies have shown that metal nanowires can be created in the pores of porous alumina using the electrodepostion method. The reason is that a non-conducting layer that exists at the interface between porous alumina and the aluminium substrate, and this is sufficiently thick that it does not allow electrons to tunnel through during electrodeposition. ${ }^{15,16}$

To get highly ordered nanoporous alumina on an aluminium substrate, a two-step anodisation process can be used that was introduced by Masuda et al. ${ }^{17}$ The geometry of porous alumina (pore diameter, interpore distance and film thickness) can be controlled by controlling the anodizing parameters such as anodising voltage, electrolyte concentration, temperature and time of anodisation. In all 
cases a non-conducting dense oxide layer called the barrier layer exists at the interface between the porous alumina and aluminium. ${ }^{18}$ This oxide layer prevents electrochemical deposition of metal. ${ }^{16,19-22}$ However previous studies have shown that the barrier layer can be reduced or thinned either by cathodic polarization ${ }^{23-25}$ or stepwise reduction in anodisation voltage/current. ${ }^{10,15,26-29}$ Cathodic polarization involves reversing the polarity in a different electrochemical bath, and electrochemically etching the barrier layer, however it not only etches the barrier layer but also etches the pore walls thickness of porous anodic alumina (PAA). ${ }^{25}$ To overcome this problem without affecting the wall thickness a stepwise voltage reduction can be used. ${ }^{10,15,26-29}$ In this method, the voltage is reduced in steps immediately after the second anodisation process. Since the geometry depends on the anodising parameters (for example pore diameter is linearly proportional to the anodising voltage) the voltage reduction process results in a dendrite like pore structure at the bottom of the main pores. ${ }^{28}$ After thinning of the barrier layer by such a process metal nanowires can be fabricated by filling metals into the pores. ${ }^{16}$

Metal nanowires can be prepared by several other methods, but pulse electrodeposition (PED) in the template is a very simple, controlled, highly repeatable and costeffective method and it also can be applied in wide range of materials. There are several studies of fabrication of nanowires such as $\mathrm{Ag}, \mathrm{Ni}, \mathrm{Zn}$ and Co nanowires have been produced using PAA template. ${ }^{16,30-32}$ Previous studies have also reported that the growth of nanowires by the electrochemical deposition method prefers certain axial crystallographic orientations which are influenced by electrochemical deposition conditions such as applied potential. The growth of metal crystallites during electrodeposition using porous materials is associated with a mobility assisted growth model that requires nucleation from the cathode substrate at the bottom of the pores. ${ }^{33}$ To date most research has focused on the ordering of metal nanowires with uniform diameter (i.e., the control of their position and spacing), deposited in porous alumina with a regular, ordered structure. However, it is also essential to control the length and diametric uniformity along the length of nanowires for applications such as transparent conducting films. In literature, a few articles have reported the fabrication of $\mathrm{Cu}$ nanowires ${ }^{34}$ but some questions still remain about how to control the length of $\mathrm{Cu}$ nanowires, their diameters and uniformity along their length as maintaining diffusion metal ions from bulk electrolyte is more challenging.

In this work a highly controlled growth of $\mathrm{Cu}$ nanowires is achieved in a PAA template formed by anodisation of pure aluminum $(\mathrm{Al})$ by creating a dendritic porous path at the interface of the PAA and the $\mathrm{Al}$ substrate during the anodisation process. Copper is then deposited by pulsed electrodeposition and after mild etching of the alumina template this results in highly aligned $\mathrm{Cu}$ nanowires. The thickness of the nanowires is controlled by the anodisation process. The ability to tune the length and thickness of such ordered copper nanowires could provide a large flexibility in material choice for transparent conducting film applications.

\section{EXPERIMENTAL DETAILS}

Copper nanowires were fabricated by filling metal into nanoporous alumina (NPA) by the pulse-electrodeposition method. The process is shown schematically in Figure 1. PAA was obtained by anodisation of pure aluminium. Pure $\mathrm{Al}$ sheet $(99.999 \%)$ with a dimension of $10 \mathrm{~mm} \times 10 \mathrm{~mm} \times$ $2 \mathrm{~mm}$ was obtained from Advent Research materials. Before anodisation the pure $\mathrm{Al}$ samples were subjected to a series of pretreatment processes. Mechanical polishing was carried out using silicon carbide paper with different grit size varying from 400 to 2500 , followed by $0.5 \mu \mathrm{m}$ diamond paste used on selvet cloth to get mirror finish surfaces. Subsequently, the samples were electrochemically polished in a solution of $20 \%$ perchloric acid in ethanol at a constant voltage of $10 \mathrm{~V}$ for 3 minutes. Initially the current density was high at $200 \mathrm{~mA} / \mathrm{cm}^{2}$. It subsequently decreased and after about $30 \mathrm{~s}$ it got stabilised at $70 \mathrm{~mA} / \mathrm{cm}^{2}$. After electropolishing, the samples were rinsed several times in DI (deionized) water. The average roughness value $\left(R_{a}\right)$ was measured using atomic force microscopy (AFM) and it was around $10 \pm 2 \mathrm{~nm}$ for a scan length of 90 microns. After the pretreatment process, the first step anodisation was carried out in a solution of $0.3 \mathrm{M} \mathrm{C}_{2} \mathrm{H}_{2} \mathrm{O}_{4}$ (oxalic acid) at constant voltage of $40 \mathrm{~V}$ at $18{ }^{\circ} \mathrm{C}$ for 30 minutes. The current density was high $\left(100 \mathrm{~mA} / \mathrm{cm}^{2}\right)$ initially, measured as soon as the samples were immersed in the electrolyte bath and it rapidly decreased to $5 \mathrm{~mA} / \mathrm{cm}^{2}$ in few second and remained constant. The non-uniform porous alumina layer which was obtained after the first anodisation, was then chemically dissolved (chemical etching) in a mixture of $6 \mathrm{wt} \% \mathrm{H}_{3} \mathrm{PO}_{4}$ (Phosphoric acid) +1.8 wt $\% \mathrm{H}_{2} \mathrm{Cr}_{2} \mathrm{O}_{4}$ (Chromic acid) at $50{ }^{\circ} \mathrm{C}$ for about 30 minutes. The dissolution time had to be precisely controlled to just etch out the porous alumina leaving dimples at the aluminium substrate. These ordered dimples formed the nucleation sites for the pores in second step anodisation. ${ }^{17}$ After chemical etching, the second anodisation was carried out under the same conditions as the first anodisation. Thereafter the barrier layer was thinned gradually by reducing the anodising voltage from $40 \mathrm{~V}$ to $0 \mathrm{~V}$ linearly in steps of $1 \mathrm{~V}$ every 15 seconds. ${ }^{35}$ After barrier layer thinning, the mild chemical etching was carried out in $0.63 \mathrm{M} \mathrm{C}_{2} \mathrm{H}_{2} \mathrm{O}_{4}$ solution at $25^{\circ} \mathrm{C}$ for $2 \mathrm{hrs}$. The samples obtained were rinsed in distilled water several times and then dried under a nitrogen purge.

The pulse electrodeposition (PED) method in a mixture of $0.5 \mathrm{M} \mathrm{Cu}_{2} \mathrm{SO}_{4}$ (copper sulphate) and $0.57 \mathrm{M} \mathrm{H}_{3} \mathrm{BO}_{3}$ (boric acid) solution was used for filling $\mathrm{Cu}$ into the pores 


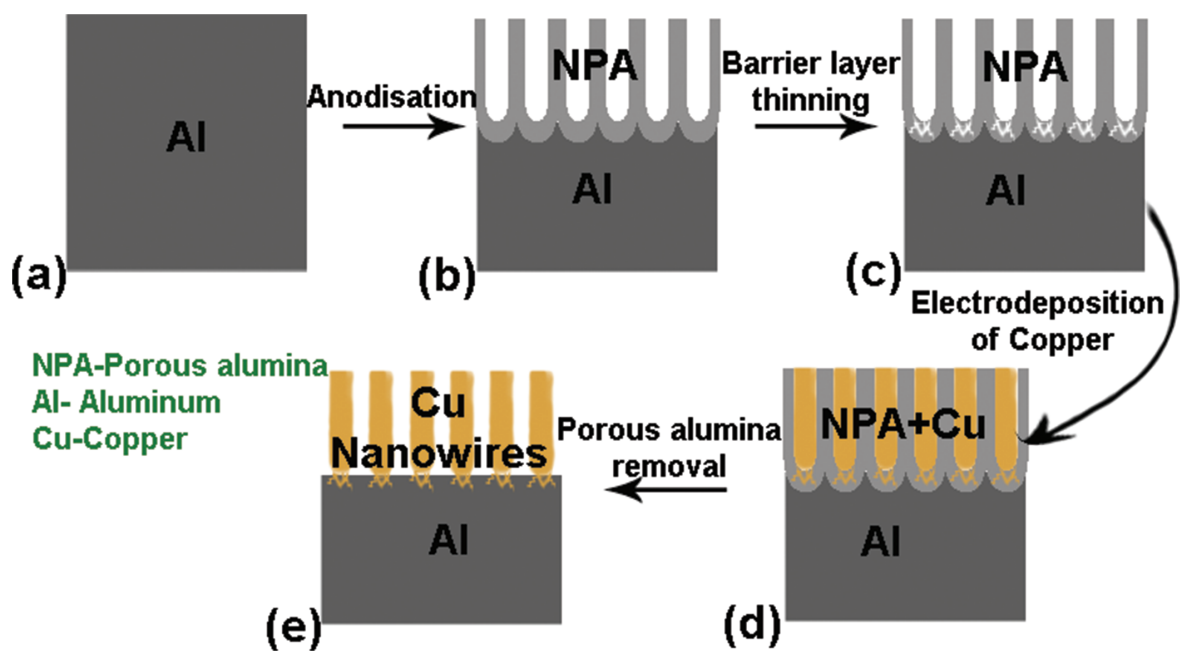

Figure 1. Schematic representation of the formation of copper nanowires (CuNWs) using a nanoporous alumina (NPA) template: (a) Electropolished pure aluminium sample (b) two-step anodisation in oxalic acid to obtain highly ordered porous alumina, (c) barrier thinning performed by reducing the voltage stepwise, (d) Cu filling into the porous alumina by pulse electrodeposition and (e) porous alumina removed to get CuNWs.

of the porous alumina template. Before electrodeposition the sample were kept in the solution for 2 hrs. Electrodeposition was carried out into the nanoporous template using $\mathrm{Pt}$ as a counter electrode. A positive pulse of $7 \mathrm{~V}$ for $3.2 \mathrm{~ms}$ and a negative pulse of $17 \mathrm{~V}$ for the duration of $3.2 \mathrm{~ms}$ were applied to deposit copper followed by a delay of $50 \mathrm{~ms}$. The delay time (electrode off) allowed for compensation of metal ion depletion near the cathode. In order to liberate formed $\mathrm{Cu}$ nanowires from the PAA membrane chemical etching in $0.1 \mathrm{M} \mathrm{NaOH}$ was performed for 2 minutes at $30{ }^{\circ} \mathrm{C}$.

The morphology of the porous alumina and $\mathrm{Cu}$ nanowires was analysed using field emission scanning electron microscopy (FE-SEM). To obtain reliable data at least a hundred nanowires were measured using Adobe Photoshop CS5 software in order to get quantitative diameter and length of nanowires. The crystal structure was characterised using X-ray powder diffraction (XRD) with $\mathrm{Cu} \mathrm{K} \alpha$ radiation $(=0.1542 \mathrm{~nm})$. XRD was conducted with sample support surface perpendicular to the length of the nanowires.

\section{RESULTS AND DISCUSSION}

A uniform NPA coating was formed using the two step anodisation procedure introduced by Masuda et al. ${ }^{17}$ The variation in current density during the anodisation using the two-step process is shown in Figure 2(a). The current density is approximately constant during the anodisation stage. The corresponding SEM images of the top surface and cross-sectional view of the porous alumina taken after 10 minutes of anodisation are also included in the figure. The dimensional parameters of the structure were calculated from the top surface and cross-sectional SEM images. The porous alumina template possesses nearly equal pore diameter of $45 \pm 5 \mathrm{~nm}$ and interpore distance of $100 \pm 5 \mathrm{~nm}$ along the pore length (Fig. 2(a)). The twodimensional ordered honeycomb porous structure can be seen from the bottom of pores. The porous oxide layer and substrate are separated by a compact oxide barrier layer.

This non-conducting barrier layer in-between the porous alumina and the aluminium substrate is then reduced using the stepwise reduction of voltage just after the

(a)
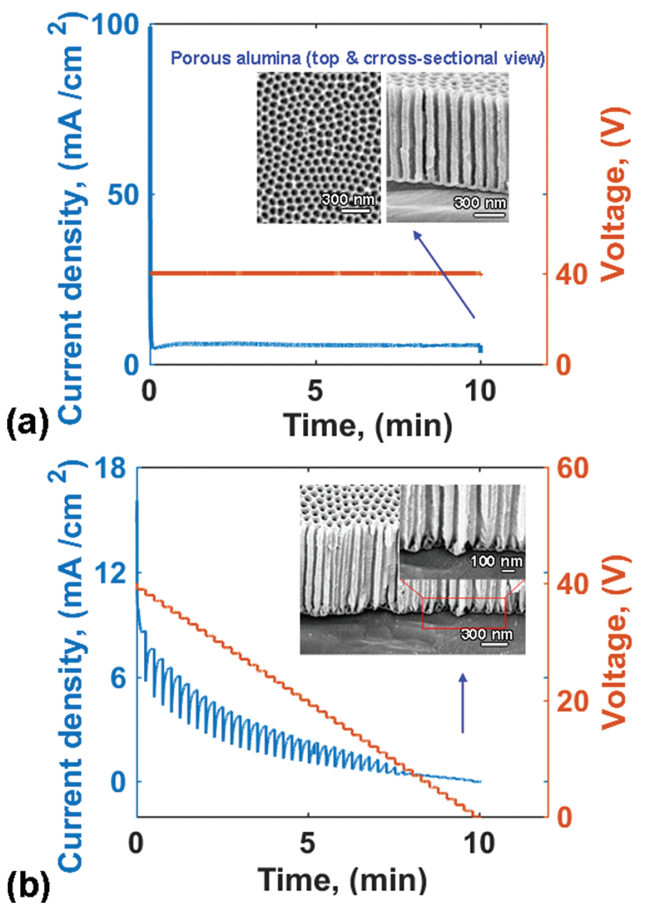

Figure 2. Current density and voltage versus time graph (a) during anodisation and corresponding SEM top and cross-sectional view of the porous alumina (b) during voltage reduction at the rate of $1 \mathrm{~V} / 15 \mathrm{~s}$ after the anodisation and SEM cross-sectional view after creating dendritic path at the bottom of the pores of the porous alumina template. 
second anodisation in the same electrolyte as shown in Figure 2(b). Yadav et $\mathrm{al}^{35}$ had found that the dendritic structure formed by reducing the voltage stepwise by $1 \mathrm{~V} / 15 \mathrm{~s}$ resulted in more than $95 \%$ of pore barrier layers being removed over a $10 \mathrm{~mm} \times 10 \mathrm{~mm}$ area. Choosing a linear reduction of voltage offers fine control over the resulting dendritic structure at the pore base. ${ }^{35}$ In order to remove the irregularities in the dendritic structure a mild chemical etching was done in $0.63 \mathrm{M} \mathrm{C}_{2} \mathrm{H}_{2} \mathrm{O}_{4}$ solution at $25{ }^{\circ} \mathrm{C}$ after the voltage reduction. This process is different from the one reported by Nielsh et al. ${ }^{16}$ wherein the pore widening is carried out before barrier thinning. We found that our procedure helped in controlling uniform deposition of $\mathrm{Cu}$ in all the pores distributed over $1 \mathrm{~cm} \times 1 \mathrm{~cm}$ area. The SEM cross-sectional view (Fig. 2(b)) confirms the thinning of the barrier layer at the bottom of the pores of the porous alumina. The shape of the thinned structure depends on the steps of potential applied during the voltage reduction. However, the kinetic control of the metal growth does not depend on the number of branches. ${ }^{31}$ It is worth noting that the diameters of the pores at the bottom are smaller, generally by few nanometres, than the diameter of the pores in rest of the coating which is due to reduction of voltage. This does not affect the subsequent metal deposition.

After the two-step anodisation and creating the conducting path at the bottom of the pores copper was filled into the pores of PAA using the pulse electrodeposition (PED) method. ${ }^{16,30,36}$ The current density measured in a single pulse during the deposition was shown in Figure 3(a). The positive pulse was applied first which resulted in copper growing uniformly in the pores. ${ }^{35}$

Initially, during the dendritic filling the current fluctuated for about $30 \mathrm{~s}$, and then the growth of CuNWs started with very little variation in the current pulse. It was observed that the current increased slightly at the start of the positive pulse and then exponentially decayed to a value of about $\sim 250 \mathrm{~mA} / \mathrm{cm}^{2}$ after less than a millisecond (Fig. 3(a)). This was, probably, because any uneven growth of the nanowires was dissolved favorably at the start of the positive pulse. ${ }^{16,37}$ During filling the deposition current remained at steady state $\left(\sim 270 \mathrm{~mA} / \mathrm{cm}^{2}\right)$ and then suddenly increased in magnitude $\left(\sim 500 \mathrm{~mA} / \mathrm{cm}^{2}\right)$ that indicated overgrowth on the surface (Fig. 3(b)).

Due to the anodizing process, vertically oriented large pores possessed a regular hexagonal pattern of cylindrical pores spread over the entire area of the porous template. ${ }^{18}$ However, during deposition, some nanowires in the template grew faster and reached the top of the template creating uneven growth on the surface. The unfilled or half-filled pores were blocked which might have been due to hydrogen bubbles and, as a result, further electrolyte transportation into the unfilled pores was terminated. Incomplete, uneven filling of pores may have happened because of the high growth rate of the
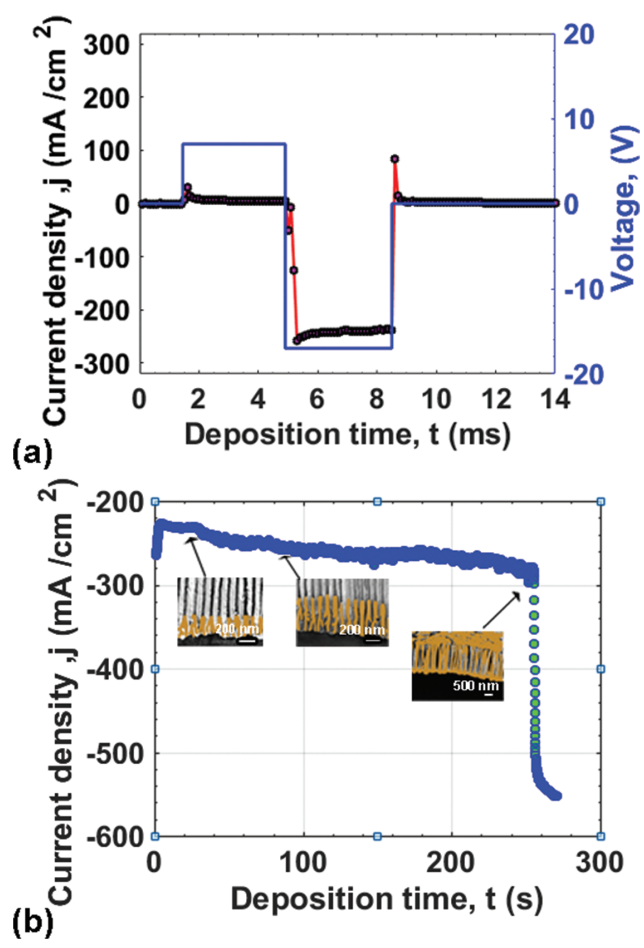

Figure 3. Current density versus time during pulsed electro deposition: (a) Current for individual pulse. (b) Variation in current density of the deposition pulse during electrodeposition. In the initial pulses all the dendrites were filled and the sudden increase in the magnitude of current indicated that the $\mathrm{Cu}$ had been filled inside the pores and overgrowth on the surface began.

nanowires. Therefore, in order to fabricate uniformly distributed nanowires and prevent uneven growth, it was essential to optimize the positive pulse during the deposition. For instance, we have found that same pulse duration with slightly lower potential than the negative pulse, could prevent the uneven growth. ${ }^{35}$ Once the porous template was filled with CuNWs, the current increased suddenly as shown in Figure 3(b). This can be used to define the end point of the nanowire deposition. By using optimized pulse conditions, it was ensured that almost all the pores were filled by $\mathrm{Cu}$. The total deposition time controls the length of the CuNWs.

Selective dissolution of alumina in $\mathrm{NaOH}$ allowed the production of free standing nanowires. Figure 4 shows SEM images of the top surfaces of CuNWs with different length obtained in this manner. All CuNWs has approximately the same diameter which did not vary systematically along the fibre length. It was observed that the diameter of the freestanding nanowire was slightly larger than the initial mean pore diameter of the template. The measured mean diameter of the CuNWs was $60 \pm 5 \mathrm{~nm}$, which was about $5 \mathrm{~nm}$ larger than the mean diameter of the pores. This was due to the widening of pore diameters of the porous alumina as it was immersed in a solution of $\mathrm{pH} \sim 4$ before and during the electrodeposition process. The $10 \mathrm{~nm}$ discrepancy was needed to be accounted for if 


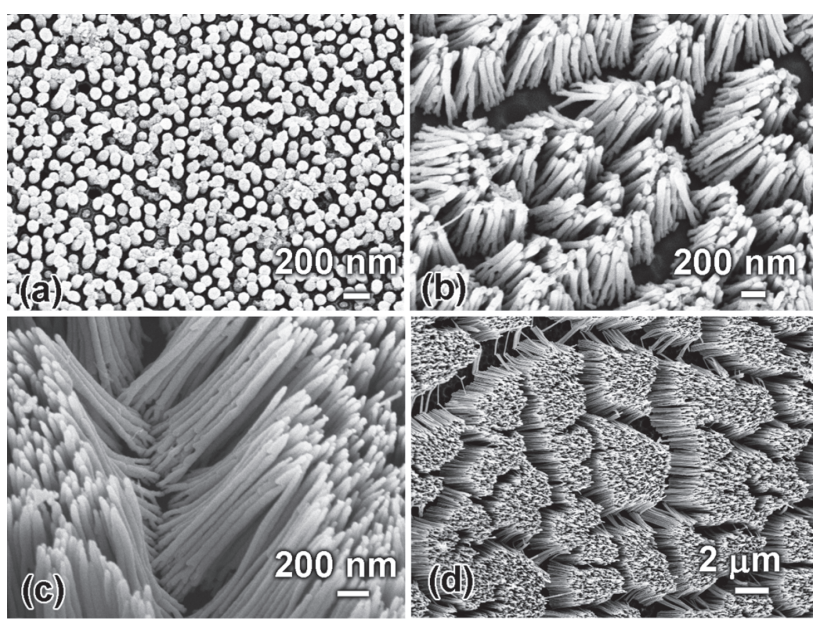

Figure 4. SEM images of CuNWs after removing the porous alumina, deposed with various time of (a) $5 \mathrm{sec}$, (b) 1 minutes, (c) 2.2 minutes, and (d) 4 minutes.

precise control of copper nanowire diameter was required. It was seen that almost all growth over a large area contained CuNWs but these were conglomerated into separate bundles. This occurred as a consequence of surface tension effects of the solution/distilled water as nanowire had been drier after the etching.

Figure 4 illustrates that the vertically aligned CuNWs lengths vary from a few nanometres to microns in size and could controlled by monitoring the current during deposition. By varying deposition current application from 5 seconds to 4 minutes the length of CuNWs can be tuned from $50 \mathrm{~nm}$ to $2 \mu \mathrm{m}$. This confirmed that the time of deposition was controlling the filling of $\mathrm{Cu}$ into the pores of alumina.

Figure 5 shows the XRD patterns of the CuNWs on the aluminum substrate formed by pulsed electrodeposition. The CuNWs have the expected fcc structure with a $\langle 111\rangle$ preferred fibre axis orientation. It has been reported that the most predominant distinct reflection in XRD pattern in $\mathrm{CuNWs}$ is $\mathrm{Cu}$ (111) when grown perpendicular

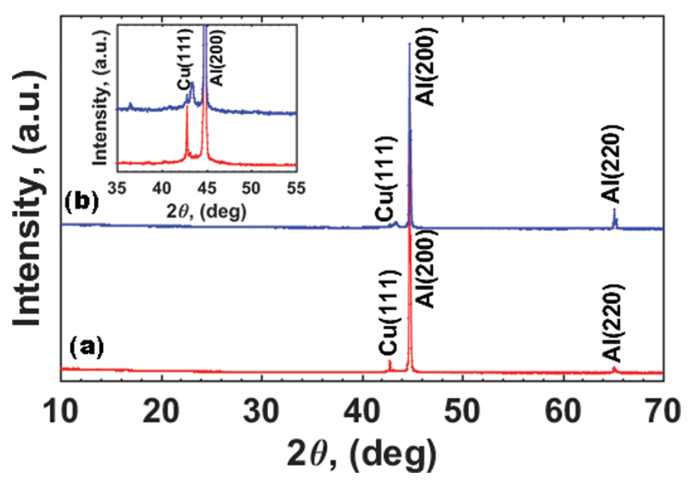

Figure 5. XRD patterns of CuNWs deposited in the porous alumina template (a) after completely removing the porous alumina (b) after partially removing the porous alumina. to a substrate surface. ${ }^{38}$ However the preferred orientation depends on the deposition conditions. ${ }^{33}$ In the case of the electrodeposition technique it has been reported that the crystallinity depends on electrodeposition parameter like deposition potential and temperature. ${ }^{33}$ By controlling the temperature and potential, single crystal of $\mathrm{Ni}$ nanowires has been produced. ${ }^{33}$ It has been also reported that the electrodepostion method can allow accomplishing the uniform preferred growth orientation by varying the applied potential. ${ }^{33}$ The same result was observed here for $\mathrm{Cu}$. Figure 5(b) shows the XRD patterns of the partially etched $\mathrm{Cu}$ NWs on the aluminum substrate. The XRD results confirmed that there was no significant difference which arises due to the alumina etching process. The large peak at $2 \theta$ value $44.7^{\circ}$ was characteristic of (200) aluminium, ${ }^{39}$ whilst the intense (111) peak of the aluminium substrate is not visible in the data due to the texturing. The major peaks of $\mathrm{Cu}$ and $\mathrm{Al}$ therefore had different orientations in Figure 5(b).

\section{CONCLUSION}

Highly aligned copper nanowires with controlled length can be fabricated by pulsed electrodeposition of $\mathrm{Cu}$ into the pores of NPA template obtained by anodisation of pure aluminum. Uniform deposition is obtained after creating the dendritic structure at the bottom of the pores through the stepwise voltage reduction method. A uniform almost $100 \%$ filling of copper is achieved after optimizing the total time of deposition. The CuNWs length was highly controlled from $100 \mathrm{~nm}-2 \mu \mathrm{m}$ with the diameter of $40 \mathrm{~nm}$ by monitoring current density during the deposition. XRD patterns confirm that the $\mathrm{Cu}$ NWs are highly oriented with axes in $\mathrm{Cu}$ [111] direction. The growth of CuNWs with controlled length and uniform diameter was obtained using porous alumina templates. This can have implications towards various nanoelectronic applications such as transparent conducting films. However, the passivation of the nanowire surface to prevent oxidation remains an issue to be solved.

Acknowledgments: The work was in part supported by Indian Space Research Organisation (ISRO).

\section{References and Notes}

1. J. Hu, T. W. Odom, and C. M. Lieber, Acc. Chem. Res. 32, 435 (1999).

2. J. D. Holmes, K. P. Johnston, R. C. Doty, and B. A. Korgel, Science 287, 1471 (2000).

3. C. M. Lieber, Solid State Commun. 107, 607 (1998).

4. S. Jewell and S. M. Kimball, Mineral Commodity Summaries 2014; US Geological Survey, Reston, VA, USA (2014).

5. F. Cui, Y. Yu, L. Dou, J. Sun, Q. Yang, C. Schildknecht, K. SchierleArndt, and P. Yang, Nano Lett. 15, 7610 (2015).

6. S. Ye, A. R. Rathmell, I. E. Stewart, Y. C. Ha, A. R. Wilson, Z. Chen, and B. J. Wiley, Chem. Commun. 50, 2562 (2014).

7. S. Han, S. Hong, J. Ham, J. Yeo, J. Lee, B. Kang, P. Lee, J. Kwon, S. S. Lee, M. Y. Yang, and S. H. Ko, Adv. Mater. 26, 5808 (2014). 
8. T. Thurn-Albrecht, J. Schotter, G. A. Kastle, N. Emley, T. Shibauchi, L. Krusin-Elbaum, K. Guarini, C. T. Black, M. T. Tuominen, and T. P. Russell, Science 290, 2126 (2000).

9. H. Park, A. K. L. Lim, A. P. Alivisatos, J. Park, and P. L. McEuen, Appl. Phys. Lett. 75, 301 (1999).

10. C. R. Martin, Chem. Mater. 8,1739 (1996)

11. G. E. Thompson and G. C. Wood, Nature 290, 230 (1981).

12. C. Schonenberger, B. M. I. van der Zande, L. G. J. Fokkink, M. Henny, C. Schmid, M. Kruger, A. Bachtold, R. Huber, H. Birk, and U. Staufer, J. Phys. Chem. B 101, 5497 (1997).

13. Y. J. Han, J. M. Kim, and G. D. Stucky, Chem. Mater. 12, 2068 (2000).

14. G. Thompson, Thin Solid Films 297, 192 (1997).

15. J. Choi, G. Sauer, K. Nielsch, R. B. Wehrspohn, and U. Gosele, Chem. Mater. 15, 776 (2003).

16. K. Nielsch, F. Muller, A. P. Li, and U. Gosele, Adv. Mater. 12,582 (2000).

17. H. Masuda and K. Fukuda, Science 268, 1466 (1995).

18. G. Thompson, Thin Solid Films 297, 192 (1997).

19. D. AlMawlawi, N. Coombs, and M. Moskovits, J. Appl. Phys. 7, 4421 (1991)

20. F. Li, R. M. Metzger, and W. Doyle, IEEE Transactions on Magnetics 33, 3715 (1997).

21. D. Routkevitch, T. Bigioni, M. Moskovits, and J. M. Xu, J. Phys. Chem. 100, 14037 (1996).

22. D. Routkevitch, J. Chan, J. Xu, and M. Moskovits, Porous anodic alumina templates for advanced nanofabrication. Proceedings of the International Symposium on Pits and Pores: Formation, Properties, and Significance for Advanced Luminescent Materials (1997), Vol. 97, pp. 350-357.

23. O. Rabin, P. R. Herz, Y. M. Lin, A. I. Akinwande, S. B. Cronin, and M. S. Dresselhaus, Adv. Funct. Mater. 13, 631 (2003).
24. M. Tian, S. Xu, J. Wang, N. Kumar, E. Wertz, Q. Li, P. M. Campbell, M. H. Chan, and T. E. Mallouk, Nano Letters 5, 697 (2005).

25. X. Zhao, S. K. Seo, U. J. Lee, and K. H. Lee, J. Electrochem. Soc. 154, 553 (2007).

26. M. A. El-Sayed, Acc. Chem. Res. 34, 257 (2001).

27. W. Xu, H. Chen, M. Zheng, G. Ding, and W. Shen, Opt. Mater. 28, 1160 (2006).

28. W. Cheng, M. Steinhart, U. Gosele, and R. B. Wehrspohn, J. Mater. Chem. 17, 3493 (2007).

29. M. Shaban, H. Hamdy, F. Shahin, J. Park, and S. W. Ryu, J. Nanosci. Nanotechnol. 10, 3380 (2010).

30. G. Sauer, G. Brehm, S. Schneider, K. Nielsch, R. B. Wehrspohn, J. Choi, H. Hofmeister, and U. Gosele, J. Appl. Phys. 91, 3243 (2002).

31. A. A. Noyan, A. P. Leontiev, M. V. Yakovlev, I. V. Roslyakov, G. A. Tsirlina, and K. S. Napolskii, Electrochim. Acta 226, 60 (2017).

32. D. Routkevitch, T. Bigioni, M. Moskovits, and J. M. Xu, J. Phys. Chem. 100, 14037 (1996).

33. S. Dellis, A. Christoulaki, N. Spiliopoulos, D. L. Anastassopoulos, and A. A. Vradis, J. Appl. Phys. 114, 164308 (2013).

34. N. J. Gerein and J. A. Haber, J. Phys. Chem. B 109, 17372 (2005).

35. A. Yadav, M. Muthukumar, and M. S. Bobji, Materials Research Express 3, 085021 (2016).

36. N. J. Gerein and J. A. Haber, J. Phys. Chem. B 109, 17372 (2005).

37. C. T. Sousa, D. C. Leitao, M. P. Proença, A. Apolinario, J. G. Correia, J. Ventura, and J. P. Araujo, Nanotechnology 22, 315602 (2011).

38. L. Zaraska, G. D. Sulka, and M. Jaskuła, Appl. Surf. Sci. 258, 7781 (2012).

39. M. Rashad, F. Pan, A. Tang, and M. Asif, Progress in Natural Science: Materials International 24, 101 (2014).

Received: 25 September 2018. Accepted: 10 January 2019. 